\title{
Delimitando el punitivismo. Las actitudes de los españoles hacia el castigo de los infractores juveniles y adultos
}

\section{Portraying punitivism. Spaniards' attitudes towards the punishment of juvenile and adult offenders}

Artículo recibido el 15 septiembre 2015/Publicado el 15 diciembre 2015

Eva Aizpurúa ${ }^{1}$

Universidad de Castilla-La Mancha

\begin{abstract}
RESUMEN
El estudio de las actitudes punitivas se ha intensificado en las últimas décadas. Sin embargo, los instrumentos empleados en su medición no han tenido una evolución semejante. El objetivo de este trabajo ha sido elaborar y validar una escala en una muestra representativa de la población española $(n=1.000,51,1 \%$ mujeres, edad media=46,3 años). Para ello se ha utilizado un diseño de validación cruzada, dividiendo la muestra en dos mitades equivalentes a las que se aplicaron análisis factoriales exploratorios y confirmatorios. Los resultados aportan evidencias de la fiabilidad y validez de la escala compuesta por dos factores de primer orden ("actitudes hacia la delincuencia” y "actitudes hacia la delincuencia juvenil”) y un factor de segundo orden. Las diferencias entre sub-escalas son significativas y la exigencia de severidad es mayor cuando los infractores son adultos. Asimismo, el análisis de conglomerados indica que la mayoría de los sujetos no apoya o rechaza todas las medidas punitivas, sino que adopta posiciones intermedias.
\end{abstract}

Palabras clave. Opinión pública, delincuencia, actitudes punitivas, justicia penal, validación de una escala.

\footnotetext{
${ }^{1}$ La correspondencia debe enviarse a: Eva Aizpurúa. Centro de Investigación en criminología. Universidad Castilla-La Mancha. Edificio Benjamín Palencia. Campus Universitario, s/n. 02071 Albacete (España). Eva.Aizpurua@uclm.es
} 


\begin{abstract}
The study of punitive attitudes has increased in recent decades. The measurement instruments to gauge them, however, have not increased in parallel. The aim of this work has been to develop and validate a scale on a representative sample of the Spanish population $(n=1,000,51.1 \%$ women, average age $=46.3)$. To this end a cross-validation design has been employed, randomly assigning the sample into two groups, to which exploratory and confirmatory factorial analyses were applied. The results provide evidence on the reliability and validity of the scale, composed of two first-order factors ("attitudes towards delinquency" and "attitudes towards juvenile delinquency") and one second-order factor. The differences between subscales are significant, with demands for severity greater when offenders are adults. Moreover, cluster analysis indicates that most of the subjects do not support or reject all punitive measures, but rather adopt intermediate positions.
\end{abstract}

Keywords. Public opinion, delinquency, punitive attitudes, criminal justice, scale validation.

\title{
1. Introducción
}

A pesar del incremento de estudios destinados a conocer las actitudes punitivas de los ciudadanos, no existe acuerdo en relación con su definición y medición (Matthews, 2005; Serrano, 2013). Como explicaba Hamilton (2014) recientemente, el término punitivismo se ha utilizado tanto para explicar la evolución de la política criminal, como para describir la opinión pública hacia el castigo. Aun considerando sólo la última acepción, por ser el objeto de estudio de este trabajo, es reseñable la diversidad de formas que se han utilizado para medir las actitudes punitivas (Adriaenssen y Aertsen, 2015; Maguire y Johnson, 2015). Esta ausencia de criterios comunes explica la variedad de indicadores que se han empleado, dando como resultado la inconsistente operacionalización del concepto. Con todo, el análisis de la literatura permite identificar cinco estrategias fundamentales:

(i) La primera de ellas ha sido evaluar el apoyo de la población hacia sanciones específicas como son la pena de muerte (Baumer, Messner y Rossenfeld, 2003; Cochran, Boots y Heide, 2003; Moon, Wright, Cullen y Pealer, 2000; O’Neil, Patry y Penrod, 2004; Vogel y Vogel, 2003), la imposición de sentencias mínimas obligatorias como la iniciativa “three strikes” (Applegate, Cullen, Turner y Sundt, 1996; Roberts, 
Crutcher y Verbrugge, 2007; Tyler y Boeckmann, 1997), la transferencia de los menores a tribunales de adultos (Aizpurúa y Fernández, 2014; Allen, Trzcinski y Kubiak, 2012; Hartnagel y Templeton, 2012; Mears, 2001; Pickett y Chiricos, 2012; Schwartz, Guo y Kerbs, 1993; Shelley, Waid y Dobbs, 2011) o la privación de ciertos derechos tras el cumplimiento de las condenas, incluyendo las restricciones residenciales y el derecho de voto (Anderson, Sample y Cain, 2015; Dawson-Edwards, 2008; Manza, Brooks y Uggen, 2004; Pickett, Mancini y Mears, 2013; Pinaire, Heumann y Bilotta, 2002).

(ii) Junto con la valoración de medidas concretas, los enunciados generales que critican la benevolencia de los tribunales y reclaman el endurecimiento de las penas han sido interpretados, también, como indicadores del punitivismo (Butter, Hermanns y Menger, 2013; Grasmick y McGill, 1994; Hogan, Chiricos y Gertz, 2005; Hohl, Stanko y Newburn, 2013; Kornhauser, 2013, 2015; Shelley et al., 2011; Warner y Davis, 2011).

(iii) Otro cuerpo de estudios ha examinado este constructo atendiendo a la finalidad de la pena. Desde esta perspectiva, las personas que privilegian el castigo, la incapacitación y la disuasión sobre otros fines han sido consideradas más punitivas (Aarten, Van Gelder, Lamet, Borgers y Van der Laan, 2015; Elffers, De Keijser, Van Koppen y Van Haeringen, 2007; Sims y Johnston, 2003; Spiranovic et al., 2012).

(iv) Un procedimiento alternativo ha sido la evaluación de las condenas como un continuo en el que la privación de libertad ha representado su extremo más punitivo y la probation su extremo menos punitivo (Applegate y Davis, 2006; Miller y Applegate, 2015; Payne, Gaine, Triplett y Danner, 2004; Sööt, 2013). En este contexto, la preferencia por el internamiento y la elección de condenas más largas han sido interpretadas como expresiones de la actitud punitiva (Evans y Adams, 2003; Varona, 2013).

(v) Finalmente, un quinto enfoque ha evaluado el posicionamiento del público hacia las condiciones de cumplimiento de las medidas. Desde esta perspectiva, el punitivismo se ha vinculado con el rechazo a que los penados accedan a determinados recursos y servicios como son los programas formativos, recreativos, de inserción laboral o de promoción de la salud (Applegate, 2001; Lenz, 2002; Hensley, Koscheski y Tewksbury, 2003; Mears, Mancini, Beaver y Gertz, 2013). 
Esta breve revisión pone de manifiesto la variedad de indicadores que se han utilizado para medir las demandas punitivas, cuyo resultado ha sido la ambigüedad teórica del concepto y la dificultad a la hora de comparar los resultados entre unos y otros estudios. Al abordar contenidos distintos, las conclusiones que se desprenden de ellos cambian notablemente, siendo en ocasiones contradictorias. Esta situación ha impedido distinguir si las diferencias en los niveles de punitivismo reflejan un desplazamiento en las preferencias del público o si, por el contrario, son el producto de un artificio metodológico (Gelb, 2008; Hutton, 2005).

Más allá de los contenidos analizados, existen dos circunstancias que explican la variabilidad de los resultados. La primera de ellas tiene que ver con los destinatarios de las medidas. Mientras que algunos trabajos han examinado la actitud hacia la delincuencia en general (Baker, Metcalfe, Berenblum, Aviv y Gertz, 2014; Butter et al., 2013; Chen y Einat, 2015; Hartnegal y Templeton, 2012; Hogan et al., 2005; Shelley et al., 2011; Welch, 2011), otros han limitado su objeto de estudio a poblaciones específicas como son los delincuentes juveniles (Baker, Cleary, Pickett y Gertz, 2013; Grasmick y McGill, 1994; Kubiak y Allen, 2011; Miller y Applegate, 2015; Mears, Pickett y Mancini, 2014; Moon et al, 2000; Sharp, McGhee, Hope y Coyne, 2007; Vogel y Vogel, 2003) o los agresores sexuales (Anderson et al., 2015; Hirsfield y Piquero, 2010; Pickett et al., 2013). Dichas investigaciones han puesto de relieve la heterogeneidad de la opinión pública, que reclama mayor severidad cuando los delitos son de naturaleza sexual y cuando sus autores son adultos (Moon et al., 2000; Pickett, Welch, Chiricos y Gertz, 2014; Rogers y Ferguson, 2011; Vogel y Vogel, 2003; Welch, 2011). La segunda razón que justifica las inconsistencias guarda relación con la dimensión de la actitud que se ha evaluado, distinguiendo entre las actitudes globales y las actitudes específicas. Las primeras permitirían esclarecer el posicionamiento general de los individuos, mientras que las segundas examinarían dicho posicionamiento bajo circunstancias particulares. En su estudio sobre opinión hacia la legislación "three strikes” Applegate y sus colaboradores (1996) encontraban que el apoyo concedido a esta iniciativa ascendía a un $90 \%$ cuando la pregunta se planteaba de forma abstracta. Sin embargo, cuando se formulaban casos concretos sólo el $17 \%$ de los participantes era favorable a ella. En la discusión de sus hallazgos, los autores destacaban la falta de correspondencia entre ambos niveles de las actitudes, recalcando la importancia del 
método en los resultados obtenidos. Aproximaciones posteriores han confirmado esta dualidad, llevando a algunos autores a distinguir entre los conceptos de aceptación (acceptance) y preferencia (preference). Esto es, las medidas que los ciudadanos estarían dispuestos a aceptar y aquellas por las que realmente optarían si tuviesen oportunidad de hacerlo (Sandys y McGarrell, 1995). En la investigación realizada por Kubiak y Allen (2011) el 42,6\% de los sujetos apoyó la cadena perpetua para los menores condenados por homicidio cuando no se proporcionaban otras opciones. No obstante, cuando se ofrecían respuestas alternativas, sólo el 5\% escogió esta pena. Turner y su equipo (1997) observaron también este fenómeno, concluyendo que la tolerancia del público era bidireccional. Tras preguntar a los encuestados las sanciones que impondrían en ciertos supuestos y analizar si aprobarían otras, encontraron que los participantes estarían dispuestos a aceptar tanto condenas más severas como condenas más benevolentes.

Todo ello pone de manifiesto la complejidad de la opinión pública hacia el delito y su control. Sin negar la existencia de rasgos punitivos, el conocimiento acumulado ha demostrado que las actitudes son, ante todo, ambivalentes y polifacéticas (Hutton, 2005). Por una parte, se aprecia cómo el apoyo hacia las políticas retributivas no es incondicional, sino que varía notablemente en función de sus destinatarios, de las características del delito y de las alternativas disponibles. Por otra parte, la literatura ha indicado que las actitudes punitivas, cuando existen, no son excluyentes de otras posturas. Recientemente, Falco y Turner (2014) advertían cómo la mayoría de los participantes consideraban que la rehabilitación de los infractores era tan importante como su castigo. Resultados similares encontraban Mears y sus colaboradores (2014) en relación con la delincuencia juvenil violenta. Aunque una parte de los encuestados avalaba un enfoque esencialmente punitivo (15\%) o rehabilitador (31\%), la mayoría de ellos apostaba por un modelo mixto (54\%). Además, en aquellos casos en los que no se ha forzado la elección de una única condena, los sujetos han preferido la combinación de penas privativas de libertad y medidas alternativas al internamiento (Jerre, 2014).

A pesar de estos avances tan significativos que han dado cuenta del carácter flexible y matizado de las actitudes, los estudios en este ámbito se enfrentan a dos limitaciones importantes que comprometen la solidez de sus resultados. La primera de ellas se refiere a su inconsistente medición. Muchos trabajos han evaluado el 
punitivismo utilizando un único indicador, con la consiguiente reducción del constructo a una sola de sus expresiones. Aun siendo una práctica habitual, diversos autores han señalado la incapacidad de las preguntas aisladas para recoger la complejidad de las actitudes, ofreciendo una representación parcial de las mismas (Hogan et al., 2005; Moon et al., 2000). Por su parte, los trabajos que han recurrido a indicadores múltiples han tendido a utilizar escalas elaboradas ad hoc cuyas propiedades métricas no han sido comprobadas empíricamente. Esta situación se agrava en España al no disponer de herramientas que hayan sido validadas y puedan aplicarse con garantías (Aizpurúa, 2014). La segunda limitación tiene que ver con el diseño muestral y hace referencia tanto a la utilización de muestras pequeñas, como al empleo de procedimientos no probabilísticos que han restringido las posibilidades de generalizar los resultados. Este trabajo pretende contribuir a superar ambas debilidades elaborando una escala que permita medir las actitudes punitivas de los españoles. Aunque se han empleado índices compuestos con anterioridad, este estudio pretende validar, por primera vez en nuestro contexto, un instrumento para medir el punitivismo utilizando una muestra representativa de la población. Adicionalmente, se propone agrupar a los sujetos según sus demandas de severidad, evaluando, asimismo, las diferencias que existen cuando los infractores son menores y adultos.

\section{Método}

\subsection{Participantes}

En el estudio han participado 1.000 sujetos residentes en España con edades comprendidas entre los dieciocho y los ochenta y ocho años, siendo su media de edad 46,33 años (DT=16,45). De ellos el 51,1\% fueron mujeres y el 48,9\% hombres.

La muestra fue extraída mediante muestreo estratificado polietápico, aplicándose cuotas de sexo y edad a las unidades últimas. Los estratos se formaron cruzando el tamaño del hábitat con la Comunidad Autónoma, distribuyéndose de manera proporcional al total de la región. Aplicando los criterios del muestreo aleatorio simple para un nivel de confianza del 95,5\% y en el supuesto de máxima indeterminación ( $\mathrm{p}=\mathrm{q}=50$ ), el error muestral se situó en $\pm 2,9 \%$. 
El trabajo de campo se realizó entre el 16 y el 21 de mayo de 2012 mediante encuesta telefónica asistida por ordenador (Computer Assited Telephone Interviewing) en el domicilio de los participantes.

\subsection{Instrumento de medida y variables empleadas}

La versión preliminar de la escala fue construida a partir de la revisión de la literatura especializada y quedó integrada por 17 indicadores medidos con escalas de once puntos en las que 0 significaba “totalmente en desacuerdo" y 10 "totalmente de acuerdo" (ver tabla 1). Se incluyeron ítems referidos a sanciones específicas $\left(X_{5}, X_{9}, X_{10}, X_{12}\right)$, al endurecimiento de las penas $\left(\mathrm{X}_{1}, \mathrm{X}_{6}, \mathrm{X}_{7}, \mathrm{X}_{14}\right)$, a las condiciones de cumplimiento de las mismas $\left(\mathrm{X}_{8}\right)$ y a los mecanismos de prevención del delito $\left(\mathrm{X}_{2}, \mathrm{X}_{3}, \mathrm{X}_{4}, \mathrm{X}_{11}, \mathrm{X}_{13}, \mathrm{X}_{15}\right.$, $\left.\mathrm{X}_{16}, \mathrm{X}_{17}\right)$. Asimismo, se evaluaron las actitudes hacia la delincuencia en general $\left(\mathrm{X}_{1}-\mathrm{X}_{9}\right)$ y hacia la delincuencia juvenil en particular $\left(\mathrm{X}_{10}-\mathrm{X}_{17}\right)$. La escala no se administró de forma independiente sino que formó parte de un cuestionario más amplio: el III Barómetro de la Actividad Judicial. 
Tabla 1. Estadísticos descriptivos de los indicadores empleados en el estudio

\begin{tabular}{|c|c|c|c|c|c|}
\hline & Variables & $\mathbf{M}$ & DT & Asim. $^{a}$ & Curt. ${ }^{b}$ \\
\hline $\mathrm{X}_{1}$ & general las penas deberían ser más duras & 7,50 & 2,708 & 056 & 553 \\
\hline $\mathbf{X}_{2}$ & $\begin{array}{l}\text { La mejor forma de prevenir la delincuencia es crear más } \\
\text { trabajo y reducir el paro }\end{array}$ & 8,07 & 2,520 & $-1,506$ & 1,989 \\
\hline $\mathbf{X}_{3}$ & $\begin{array}{l}\text { Deberían invertirse más recursos orientados a la } \\
\text { rehabilitación de los delincuentes }\end{array}$ & 6,72 & 2,871 & $-0,777$ & $-0,013$ \\
\hline $\mathbf{X}_{4}$ & Debería haber mayor control policial en las calles & 7,84 & 2,432 & $-1,202$ & 1,202 \\
\hline $\mathbf{X}_{5}$ & $\begin{array}{l}\text { Debería ser posible la cadena perpetua para los delitos de } \\
\text { especial gravedad }\end{array}$ & 7,55 & 3,350 & $-1,221$ & 0,151 \\
\hline$X_{6}$ & $\begin{array}{l}\text { La sociedad tiende a ser demasiado permisiva con los } \\
\text { delincuentes }\end{array}$ & 6,72 & 2,915 & $-0,647$ & $-0,381$ \\
\hline $\mathbf{X}_{7}$ & $\begin{array}{l}\text { En España los delincuentes no son tratados con la dureza } \\
\text { que merecen }\end{array}$ & 7,45 & 2,921 & $-1,134$ & 0,451 \\
\hline $\mathbf{X}_{8}$ & $\begin{array}{l}\text { Las personas que están en prisión deben contar con } \\
\text { instalaciones como gimnasios o bibliotecas }\end{array}$ & 4,97 & 3,352 & $-0,149$ & $-1,119$ \\
\hline $\mathrm{X}_{9}$ & $\begin{array}{l}\text { La cárcel es una pena demasiado dura y debería utilizarse } \\
\text { lo menos posible }\end{array}$ & 3,99 & 3,305 & 0,287 & $-1,096$ \\
\hline $\mathbf{X}_{10}$ & $\begin{array}{l}\text { Debería ser posible que los menores que han cometido } \\
\text { delitos muy graves fuesen enjuiciados en tribunales de } \\
\text { adultos }\end{array}$ & 7,93 & 2,828 & ,442 & 1,236 \\
\hline$X_{11}$ & $\begin{array}{l}\text { La única forma de evitar la reincidencia de los jóvenes es } \\
\text { castigarles debidamente }\end{array}$ & 6,94 & 2,934 & $-0,784$ & $-0,189$ \\
\hline$X_{12}$ & $\begin{array}{l}\text { Encarcelar a los jóvenes no tiene sentido ya que las } \\
\text { prisiones son escuelas de delincuencia }\end{array}$ & 6,34 & 3,038 & $-0,583$ & $-0,479$ \\
\hline$X_{13}$ & $\begin{array}{l}\text { La única forma de proteger a la sociedad es enviar a los } \\
\text { jóvenes a prisión y mantenerlos allí }\end{array}$ & 5,07 & 3,203 & $-0,086$ & $-0,965$ \\
\hline $\mathrm{X}_{14}$ & $\begin{array}{l}\text { Deberían endurecerse las penas para la mayoría de los } \\
\text { delitos cometidos por jóvenes }\end{array}$ & 7,02 & 2,871 & 44 & $-0,020$ \\
\hline$X_{15}$ & $\begin{array}{l}\text { Debería proporcionarse más apoyo a la familia de los } \\
\text { delincuentes juveniles }\end{array}$ & 6,45 & 2,847 & $-0,679$ & $-0,121$ \\
\hline$X_{16}$ & $\begin{array}{l}\text { Una forma de prevenir la delincuencia juvenil es reforzar } \\
\text { la disciplina y, si es necesario, la mano dura }\end{array}$ & 6,85 & 2,998 & $-0,796$ & $-0,246$ \\
\hline$X_{17}$ & $\begin{array}{l}\text { Una forma de prevenir la delincuencia juvenil es dedicar } \\
\text { más recursos a los centros escolares }\end{array}$ & 8,25 & 2,251 & $-1,648$ & 2,880 \\
\hline
\end{tabular}

${ }^{a}$ Error típico de asimetría (E.T.) 0,077.

${ }^{\mathrm{b}}$ Error típico de curtosis (E.T.) 0,155.

\subsection{Análisis de los datos}

Con la finalidad de obtener evidencias sobre la validez cruzada de la escala, la muestra total de 1.000 sujetos fue dividida aleatoriamente en dos mitades equivalentes (cada una de ellas compuesta por $500 \operatorname{casos}^{2}$ ).

\footnotetext{
${ }^{2}$ No se hallaron diferencias estadísticamente significativas entre una y otra muestra en ninguna de las variables estudiadas.
} 


\section{Sub-muestra 1}

En primer lugar se calcularon los estadísticos descriptivos y se realizaron pruebas de asociación entre las variables, descartando los ítems cuyo índice de discriminación no superó el umbral de 0,30. Posteriormente se realizó un análisis factorial exploratorio con el método de extracción de rangos mínimos (Ten Berge y Kiers, 1991) y rotación Promin (Lorenzo-Seva, 1999). Los procedimientos para estimar el número de factores fueron la implementación óptima del análisis paralelo (Horn, 1965; Timmerman y Lorenzo-Seva, 2011), el test de mínima correlación parcial promediada (MAP, Velicer, 1976) y las correlaciones residuales. Teniendo en cuenta las características de los indicadores -escalas ordinales y distribuciones alejadas de la normalidad- el análisis se realizó a partir de la matriz de correlaciones policóricas (Hair, Anderson, Tatham y Black, 2007). Finalmente, se evaluó la consistencia interna de los factores con el coeficiente alfa de Cronbach.

\section{Sub-muestra 2}

Con el objetivo de evaluar la solución inicial se llevó a cabo un análisis factorial confirmatorio con la sub-muestra 2. Considerando el incumplimiento del supuesto de normalidad, los parámetros fueron estimados con el procedimiento de distribución libre ${ }^{3}$ (Asymptotically Distribution Free). Siguiendo las recomendaciones de la literatura $(\mathrm{Hu}$ y Bentler, 1999; Tanaka, 1993), el ajuste de los modelos fue evaluado utilizando varios indicadores para garantizar la aceptabilidad de los resultados desde distintas perspectivas. La bondad del ajuste global se evaluó a través del estadístico ji-cuadrado dividido entre sus grados de libertad ( $\left.\mathrm{x}^{2} / \mathrm{gl}\right)$, el índice de bondad de ajuste (GFI), el error cuadrático medio de aproximación (RMSEA), el índice de validación cruzada esperada (ECVI), los índices de bondad de ajuste corregido (AGFI) e incremental (IFI), el índice de ajuste normado de parsimonia (PNFI) y el criterio de información de akaike (AIC). La fiabilidad fue analizada a través de los índices de fiabilidad compuesta (IFC) y varianza extraída (IVE).

\footnotetext{
${ }^{3}$ La asignación de una escala de medida a las variables latentes se llevó a cabo fijando a una constante (1) la saturación $(\lambda)$ del primer indicador de cada escala. Además, en el modelo jerárquico se fijó a 1 el coeficiente de regresión del primer factor de primer orden (Rial, Varela, Abalo y Levy, 2006).
} 


\section{Muestra Global}

Una vez validada la escala global se estudiaron las diferencias entre sub-escalas utilizando la prueba de los signos de Wilcoxon. Asimismo, con el propósito de clasificar a los participantes en función de sus demandas punitivas se aplicó un análisis de conglomerados en dos fases. La variable de clasificación fue la puntuación factorial obtenida por cada sujeto en la escala, la medida de similitud fue la distancia euclídea y el método de conglomeración fue el criterio de información de akaike (AIC).

\section{Resultados}

\subsection{Las dimensiones del punitivismo. Análisis Factorial Exploratorio (sub- muestra 1)}

En un primer momento se examinaron las características de las variables, eliminando aquellas cuyo índice de discriminación no superó el umbral de 0,30 $\left(\mathrm{r}_{\mathrm{it}}<0,30\right)$. Tras hacerlo, el inventario quedó integrado por diez indicadores $\left(\mathrm{X}_{1}, \mathrm{X}_{4}, \mathrm{X}_{5}, \mathrm{X}_{6}, \mathrm{X}_{7}, \mathrm{X}_{10}\right.$, $\mathrm{X}_{11}, \mathrm{X}_{13}, \mathrm{X}_{14} \mathrm{y} \mathrm{X}_{16}$ ) cuyas distribuciones resultaron ser asimétricamente negativas, con una desviación importante de la normalidad.

Para identificar la estructura subyacente a este conjunto de variables se llevó a cabo un análisis factorial exploratorio. El test de esfericidad de Bartlett $\left(\mathrm{X}^{2}{ }_{\text {Bartlett }}=1554,5\right.$; $\mathrm{gl}=45 ; \mathrm{p}=0,000)$, el coeficiente Kaiser-Meyer-Olkim (KMO=0,8817) y el determinante de la matriz de correlaciones (determinante $=0,043$ ) asumieron valores adecuados, indicando que los datos eran apropiados para su estudio factorial.

En la tabla 2 se muestran los resultados de las dos primeras pruebas para estimar el número de factores. El análisis paralelo optimizado aconsejó la retención de un único factor, pues sólo en él los datos empíricos explicaron una mayor proporción de varianza que aquellos generados aleatoriamente (52,0\%, en contraste con 22,9\%). El procedimiento de mínima correlación promediada apoyó también la solución unifactorial, sugiriendo que la escala mediría una única variable latente que explicaría el $71,09 \%$ de la varianza común y presentaría una elevada consistencia interna $(\alpha=0,848)$. 
Tabla 2. Análisis paralelo y prueba de mínima correlación parcial promediada

\begin{tabular}{|c|c|c|c|c|}
\hline \multicolumn{4}{|c|}{$\%$ de varianza actual y aleatoriamente obtenida } & \multirow{2}{*}{$\begin{array}{c}\text { MAP } \\
\text { Promedio parcial }\end{array}$} \\
\hline Factor & Datos observados & Datos aleatorios & Percentil 95 & \\
\hline 1 & 52,0 & 22,9 & 27,5 & 0,028 \\
\hline 2 & 12,0 & 17,4 & 20,3 & 0,056 \\
\hline 3 & 8,5 & 14,9 & 17,0 & 0,189 \\
\hline 4 & 6,9 & 12,6 & 14,4 & 1,426 \\
\hline 5 & 5,5 & 10,5 & 12,2 & 0,999 \\
\hline 6 & 5,2 & 8,5 & 10,5 & \\
\hline 7 & 4,3 & 6,5 & 8,6 & \\
\hline 8 & 3,6 & 4,4 & 6,5 & \\
\hline 9 & 1,9 & 2,3 & 4,4 & \\
\hline 10 & 0,0 & 0,0 & 0,0 & \\
\hline
\end{tabular}

Aunque los métodos de estimación fueron consistentes y sus resultados satisfactorios (las saturaciones de los indicadores fueron elevadas, oscilando entre 0,484 y 0,774 ), dado que el análisis paralelo y el procedimiento MAP no permiten distinguir entre varianza común y varianza de error (Ferrando y Anguiano, 2010), se valoró si el número de factores era adecuado explorando también las correlaciones residuales. La razón del residuo cuadrático medio $(\mathrm{RMSR}=0,069)$ no superó el criterio de Kelley (Harman, 1976) por el cual un valor de 0,0448 sería el límite máximo para considerar aceptable el modelo. De hecho, que este índice fuese mayor indica que habiendo eliminado la influencia del factor aún quedan relaciones sistemáticas por explicar, pudiendo ser conveniente extraer una segunda dimensión. Por esta razón, se replicó el análisis reteniendo dos factores. En la nueva solución la varianza común explicada se incrementó un 11,848\%. El factor 1 “actitudes hacia la delincuencia” agrupó a aquellos ítems que recogían demandas generales de rigor punitivo $\left(\mathrm{X}_{1}, \mathrm{X}_{4}, \mathrm{X}_{5}, \mathrm{X}_{6}, \mathrm{y} \mathrm{X}_{7}\right)$, mientras que el factor 2 “actitudes hacia la delincuencia juvenil” quedó conformado por las medidas dirigidas, específicamente, al control de la delincuencia juvenil $\left(\mathrm{X}_{10}, \mathrm{X}_{11}\right.$, $\mathrm{X}_{13}, \mathrm{X}_{14} \mathrm{y}_{\mathrm{16}}$ ). Los índices de simplicidad de Bentler (1977) y de sencillez de carga (Lorenzo-Seva, 2003) adoptaron valores de 0,985 y 0,500, mostrando que cada elemento se debió principalmente a una dimensión y que el modelo bifactorial presentaba una elevada simplicidad. Únicamente la variable $\mathrm{X}_{10}$ (transferencia de los 
jóvenes que han cometido delitos graves a tribunales ordinarios) mostró una estructura compleja, relacionándose con ambos factores (siendo sus pesos factoriales de 0,309 y 0,424 en uno y otro caso). La correlación entre sub-escalas fue elevada $\left(\mathrm{r}_{\mathrm{xy}}=0,711\right)$, indicando que las dimensiones estaban fuertemente relacionadas.

Tabla 3. Modelo factorial oblicuo de la sub-muestra $1 *$

\begin{tabular}{|c|c|c|c|c|}
\hline & \multicolumn{2}{|c|}{ Matriz configuración } & \multicolumn{2}{|c|}{ Matriz estructura } \\
\hline & Factor 1 & Factor 2 & Factor 1 & Factor 2 \\
\hline $\mathbf{X}_{1}$ & $-0,056$ & 0,782 & 0,501 & 0,742 \\
\hline $\mathbf{X}_{4}$ & 0,156 & 0,519 & 0,526 & 0,630 \\
\hline$X_{5}$ & 0,158 & 0,575 & 0,567 & 0,687 \\
\hline $\mathbf{X}_{6}$ & 0,008 & 0,608 & 0,441 & 0,614 \\
\hline $\mathbf{X}_{7}$ & $-0,288$ & 1,069 & 0,472 & 0,864 \\
\hline $\mathbf{X}_{10}$ & 0,309 & 0,424 & 0,610 & 0,644 \\
\hline$X_{11}$ & 0,742 & $-0,009$ & 0,735 & 0,518 \\
\hline $\mathbf{X}_{13}$ & 0,766 & $-0,206$ & 0,619 & 0,338 \\
\hline $\mathrm{X}_{14}$ & 0,671 & 0,185 & 0,802 & 0,662 \\
\hline$X_{16}$ & 0,574 & 0,072 & 0,625 & 0,480 \\
\hline Valor propio & \multicolumn{2}{|c|}{$\mathrm{F} 1=4,341$} & \multicolumn{2}{|c|}{$\mathrm{F} 2=0,729$} \\
\hline Varianza explicada & \multicolumn{2}{|c|}{$F 1=71,012 \%$} & \multicolumn{2}{|c|}{$\mathrm{F} 2=11,925 \%$} \\
\hline$\alpha$ de Cronbach & \multicolumn{2}{|c|}{$\mathrm{F} 1=0,787$} & \multicolumn{2}{|c|}{$\mathrm{F} 2=0,759$} \\
\hline
\end{tabular}

*Las saturaciones superiores a 0,30 se remarcan en negrita.

Los coeficientes de fiabilidad fueron adecuados $\left(\alpha_{\mathrm{F} 1}=0,787 \alpha_{\mathrm{F} 2}=0,759\right)$, sobre todo valorando el reducido número de ítems que contiene cada factor. Finalmente se comprueba que el valor de RMSR para este modelo es de 0,0397, satisfaciéndose el criterio de Kelley que fijaba en 0,0448 su valor máximo aceptable. 


\subsection{Análisis Factorial Confirmatorio (sub-muestra 2)}

Partiendo de los resultados obtenidos en la primera fase se realizó un análisis factorial confirmatorio en la sub-muestra 2, evaluando tres modelos alternativos:

(i) Una solución unifactorial en la que todas las variables se relacionaban con un solo factor,

(ii) Un modelo bifactorial en el que los indicadores se agrupaban en dos factores correlacionados, y

(iii) Un tercer modelo en el que los dos factores de primer orden convergían en un factor de orden superior.

La tabla 4 muestra los índices de bondad de ajuste para los tres modelos propuestos. En todos los casos las medidas de ajuste absoluto e incremental superaron los límites comúnmente admitidos $\left(\mathrm{x}^{2} / \mathrm{gl}<3\right.$; GFI $>0,90$ y $\left.\mathrm{RMSEA}<0,06\right)$, o se aproximaron a ellos (AGFI>0,90 e IFI>0,90). No obstante, el modelo unifactorial fue el que obtuvo peores resultados. Además, el índice de validación cruzada (ECVI) indicó su menor replicabilidad y las medidas de parsimonia (PNFI y AIC) fueron favorables a los modelos alternativos.

Tabla 4. Ajuste absoluto de los modelos

\begin{tabular}{|l|c|c|c|c|c|c|c|c|}
\hline & \multicolumn{4}{|c|}{ Absoluto } & \multicolumn{3}{c|}{ Incremental } & Parsimonia \\
\cline { 2 - 10 } & $\mathbf{x}^{2} /$ gl & GFI & RMSEA & ECVI & AGFI & IFI & PNFI & AIC \\
\hline $\begin{array}{l}\text { Modelo } \\
\text { unifactorial }\end{array}$ & 2,76 &, 919 &, 059 &, 274 &, 872 &, 784 &, 543 & 136,5 \\
\hline $\begin{array}{l}\text { 2 factores } \\
\text { correlacionados }\end{array}$ & 2,44 &, 930 &, 054 &, 250 &, 887 &, 829 &, 560 & 124,9 \\
\hline $\begin{array}{l}\text { 1 factor de 2 } \\
\text { orden }\end{array}$ & 2,44 &, 930 &, 054 &, 250 &, 887 &, 829 &, 560 & 124,91 \\
\hline
\end{tabular}

En las tres soluciones las cargas factoriales fueron significativas (con un nivel de confianza del 95\%), aunque fueron más altas en los modelos 2 y 3, ambos con iguales valores, lo cual reforzó su superioridad frente al modelo unifactorial (ver tabla 5). La fiabilidad de los indicadores fue modesta, aunque nuevamente los resultados de los 
modelos bifactorial y jerárquico fueron mejores, tal y como demuestra la tabla 5. En ellos, los factores explicaron entre el 26,6\% y el 73,9\% de la varianza de cada indicador. Con respecto a la fiabilidad de los constructos, tanto la escala global como las subescalas tuvieron una consistencia interna elevada y los índices de fiabilidad compuesta y varianza extraída rebasaron los niveles críticos de 0,70 y 0,50 (Hair et al., 2007).

Tabla 5. Coeficientes de determinación, fiabilidad compuesta y varianza extraída

\begin{tabular}{|c|c|c|c|c|c|c|}
\hline & Factor & Ítem & Pond. Estand. & $\mathbf{r}^{2}$ & IFC & AVE \\
\hline \multirow{10}{*}{$\begin{array}{c}\text { Modelo } \\
\text { unifactorial }\end{array}$} & \multirow{10}{*}{$\begin{array}{l}\text { Actitudes } \\
\text { punitivas }\end{array}$} & $X_{1}$ & 0,654 & 0,428 & \multirow{10}{*}{0,97} & \multirow{10}{*}{0,77} \\
\hline & & $\mathbf{X}_{4}$ & 0,498 & 0,248 & & \\
\hline & & $X_{5}$ & 0,563 & 0,317 & & \\
\hline & & $X_{6}$ & 0,626 & 0,392 & & \\
\hline & & $\mathbf{X}_{7}$ & 0,690 & 0,477 & & \\
\hline & & $X_{10}$ & 0,581 & 0,337 & & \\
\hline & & $X_{11}$ & 0,784 & 0,615 & & \\
\hline & & $\mathrm{X}_{13}$ & 0,659 & 0,435 & & \\
\hline & & $X_{14}$ & 0,850 & 0,723 & & \\
\hline & & $X_{16}$ & 0,603 & 0,364 & & \\
\hline \multirow{6}{*}{$\begin{array}{c}2 \text { factores } \\
\text { correlacionados }\end{array}$} & \multirow{5}{*}{$\begin{array}{c}\text { Actitudes } \\
\text { hacia la } \\
\text { delincuencia }\end{array}$} & $\mathrm{X}_{1}$ & 0,661 & 0,437 & \multirow{5}{*}{0,93} & \multirow{5}{*}{0,73} \\
\hline & & $X_{4}$ & 0,516 & 0,266 & & \\
\hline & & $X_{5}$ & 0,587 & 0,344 & & \\
\hline & & $\mathbf{X}_{6}$ & 0,639 & 0,408 & & \\
\hline & & $\mathbf{X}_{7}$ & 0,707 & 0,499 & & \\
\hline & \multirow{5}{*}{$\begin{array}{c}\text { Actitudes } \\
\text { hacia la } \\
\text { delincuencia } \\
\text { juvenil }\end{array}$} & $X_{10}$ & 0,574 & 0,329 & \multirow{5}{*}{0,96} & \multirow{5}{*}{0,84} \\
\hline \multirow{4}{*}{$\begin{array}{c}1 \text { factor de } 2^{o} \\
\text { orden }\end{array}$} & & $X_{11}$ & 0,787 & 0,620 & & \\
\hline & & $\mathbf{X}_{13}$ & 0,697 & 0,461 & & \\
\hline & & $X_{14}$ & 0,860 & 0,739 & & \\
\hline & & $X_{16}$ & 0,609 & 0,371 & & \\
\hline
\end{tabular}

En el modelo 2 los factores estuvieron muy relacionados entre sí $(\phi=0,862)$. Este resultado coincide con el análisis exploratorio, demostrando que las dimensiones del punitivismo no son independientes sino que la exigencia de severidad en el tratamiento de la delincuencia general guarda relación con la exigencia de severidad en el tratamiento de la delincuencia juvenil. De hecho, la elevada correlación entre ellos avala la existencia de un factor de segundo orden (punitivismo), que viene expresado en el tercer modelo. En él los parámetros fueron muy elevados, indicando una fuerte relación entre el punitivismo y los factores “actitudes hacia la delincuencia" ( $\gamma=0,911$; $\left.\mathrm{R}^{2}=0,830\right)$ y “actitudes hacia la delincuencia juvenil” $\left(\gamma=0,946 ; \mathrm{R}^{2}=0,895\right)$. 
Tomados conjuntamente, los resultados muestran que el modelo jerárquico recogido en la figura 1 es el que mejor se adecua a los datos. En contraste, el modelo unifactorial tuvo un ajuste muy pobre y presentó una menor parsimonia.

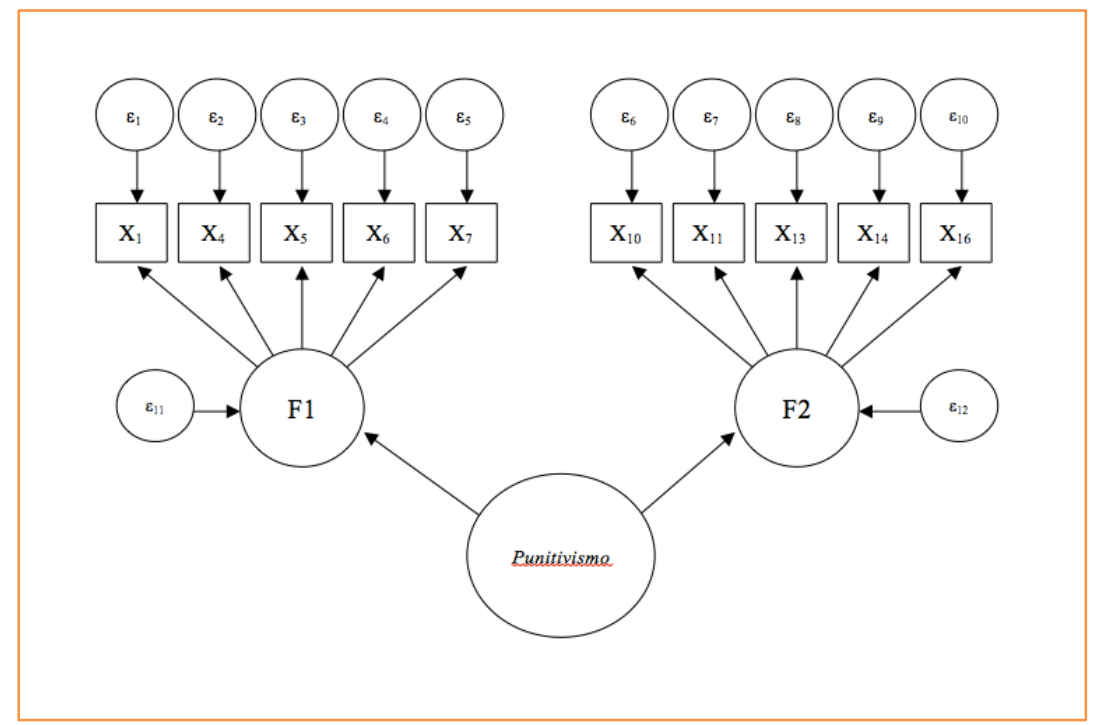

Figura 1. Diagrama estructural del modelo jerárquico con un factor de segundo orden (punitivismo) y dos factores de primer orden $\left(\mathrm{F}_{1}=\right.$ actitudes hacia la delincuencia $\mathrm{y}$ $\mathrm{F}_{2}=$ actitudes hacia la delincuencia juvenil).

\subsection{La minoría de edad, ¿Un factor corrector del punitivismo?}

Una vez validada la escala global se evaluaron sus dimensiones tratando de determinar en qué medida difieren las demandas rigoristas cuando sus destinatarios son menores o adultos. Para ello se construyeron ambas sub-escalas a partir de las puntuaciones medias de sus variables.

En ambos casos los datos recorrieron toda la métrica de las escalas (0-10) habiendo quienes rechazaron y quienes apoyaron la totalidad de las medidas. Tanto una como otra presentaron asimetría negativa, aunque las diferencias fueron notables. Como muestra la figura 2, la distribución de la escala "actitudes hacia la delincuencia” trazó una curva en forma de J, existiendo una gran concentración de valores en su extremo superior, que expresa acuerdo con las medidas propuestas y, por tanto, implica un mayor sentimiento punitivo. En cambio, en la escala "actitudes hacia la delincuencia juvenil" los datos se distribuyeron más uniformemente, y la tendencia a apoyar distintas iniciativas punitivas fue menos acentuada. 


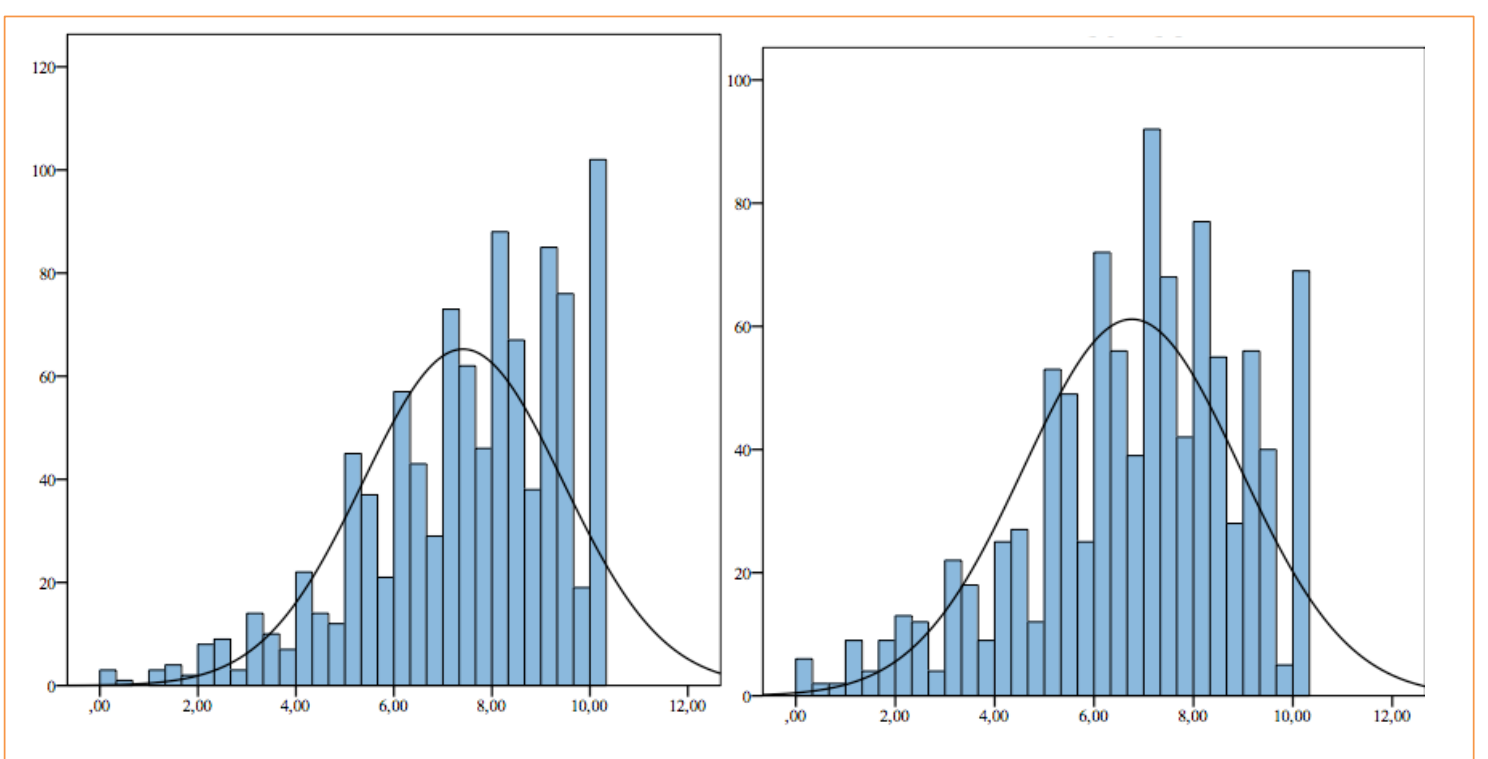

Figura 2. Histograma con curva normal de las escalas actitudes hacia la delincuencia (izquierda) y actitudes hacia la delincuencia juvenil (derecha).

Con el propósito de confirmar empíricamente estas diferencias, se aplicó el test de los rangos con signo de Wilcoxon para muestras relacionadas ${ }^{4}$. Los resultados obtenidos $(\mathrm{z}=-11,405 ; \mathrm{p}=0,000)$ demostraron que las demandas de severidad no eran iguales para los infractores juveniles y adultos, sino que la población reclamaba respuestas menos severas cuando los destinatarios eran menores. El promedio de esta escala fue 6,76 $(\mathrm{DT}=2,17)$, mientras que en la primera ascendió a 7,41 (DT=2,04). Asimismo, hubo 606 casos en los que el valor de la escala “actitudes hacia la delincuencia” fue mayor que el valor de la escala “actitudes hacia la delincuencia juvenil” y sólo 307 en los que ocurrió lo contrario.

\subsection{EI punitivismo como continuo}

Con la finalidad de clasificar a los individuos según sus demandas punitivas se aplicó un análisis de conglomerados sobre las puntuaciones factoriales de los sujetos en el factor de segundo orden $^{5}$. Sus resultados revelaron la existencia de tres grupos

\footnotetext{
${ }^{4}$ Se escogió un test no paramétrico al incumplirse el supuesto de normalidad univariante (sub-escala 1 : $\mathrm{K}-\mathrm{S}=0,102$; $\mathrm{p}=0,000$; sub-escala 2: $\mathrm{K}-\mathrm{S}=0,073 ; \mathrm{p}=0,000$ ).

${ }^{5}$ Las puntuaciones factoriales se estimaron con el método de Bartlett, realizando un análisis factorial de segundo orden a partir de las puntuaciones factoriales de las dos escalas.
} 
diferenciados ${ }^{6}$ cuya representación gráfica se muestra en la figura 3. De acuerdo con esta solución se encontró un primer grupo que integraba al $41,0 \%$ de la muestra. Su puntuación media fue -0,12 y representó a los individuos menos punitivos. El segundo conglomerado agrupó al 37,8\% de los participantes. Su media ascendió a 0,67 y reunió a un sector moderadamente punitivo, que apoyaba con más intensidad las iniciativas propuestas. El último grupo aglutinó al 21,2\% de los encuestados. Su centro se situó en 1,58 y representó al segmento más punitivo de la población que avalaba la práctica totalidad de las medidas.

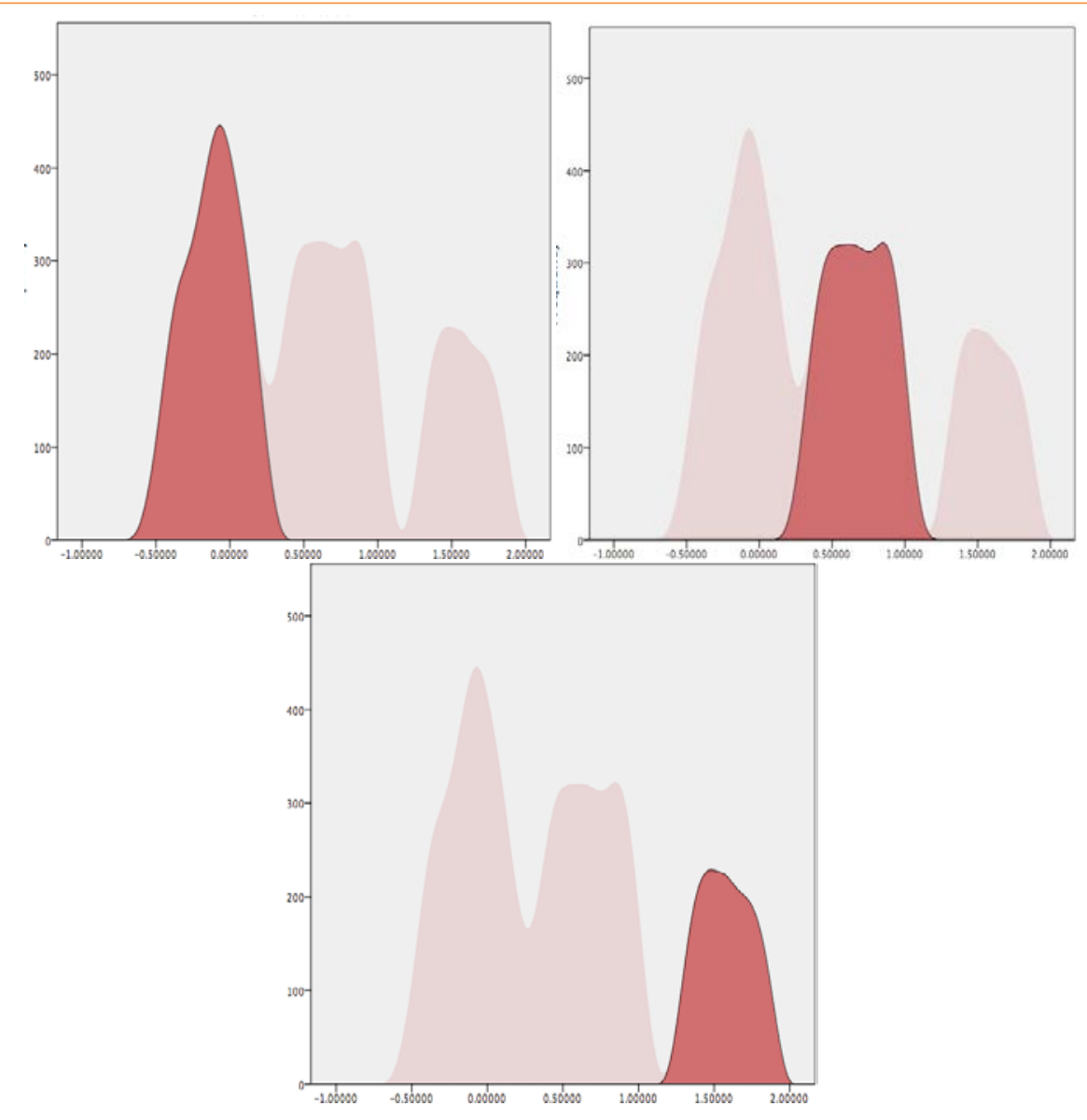

Figura 3. Distribución de los conglomerados

\footnotetext{
${ }^{6}$ La medida de silueta de cohesión y separación $(0,8)$ indicó una calidad adecuada de los conglomerados.
} 


\section{Discusión y conclusiones}

\subsection{Discusión}

Las reformas que han ido endureciendo la justicia penal han sido explicadas, en buena medida, apelando al clamor popular (Roberts y Hastings, 2007; Wood, 2009). No obstante, la diversidad de resultados que arroja la literatura y su dependencia de la metodología empleada cuestionan la imagen de una ciudadanía exclusivamente punitiva, poniendo en duda la correspondencia de las políticas impulsadas con la voluntad de los ciudadanos (Indermaur, 2009). En tales circunstancias, evaluar la existencia y la magnitud de ese desajuste constituye una labor prioritaria para la Criminología. Satisfacer este objetivo requiere llevar a cabo investigaciones que permitan conocer, con rigurosidad, las actitudes hacia la delincuencia y su control. Con esa finalidad se gestó este trabajo cuyo propósito era contribuir a la delimitación conceptual del punitivismo, superando la carencia de instrumentos cuyas propiedades hubiesen sido validadas y pudiesen aplicarse con garantías en el contexto español.

Para ello se pusieron a prueba tres modelos alternativos. El primero formado por un único factor, el segundo integrado por dos factores correlacionados y un tercer modelo en el que los dos factores de primer orden convergían en otro de orden superior. Los resultados obtenidos mostraron que los modelos bifactoriales que distinguen las actitudes hacia la delincuencia general de aquellas referidas, específicamente, a la delincuencia juvenil, se ajustan mejor a los datos. En concreto, la estructura jerárquica ofreció el ajuste más satisfactorio. En ella, los dos factores de primer orden (“actitudes hacia la delincuencia” y “actitudes hacia la delincuencia juvenil”) quedaron integrados en un factor general que representaba el punitivismo. Se obtuvieron evidencias de la validez y la fiabilidad del instrumento, cuyos pesos factoriales fueron elevados $(\lambda>0,50$; $\gamma>0,90)$ y tanto las sub-escalas como la escala global presentaron una consistencia interna adecuada.

De los análisis realizados se desprenden, además, dos hallazgos que reafirman la complejidad de la opinión pública hacia el castigo y cuestionan la interpretación uniforme que en ocasiones se ha hecho de ella. En primer lugar se aprecia como las demandas punitivas no son homogéneas, sino que varían en función de la edad de los 
infractores. En efecto, las exigencias de severidad fueron mayores cuando los delincuentes eran adultos. Estos resultados coinciden con un cuerpo de estudios que ha demostrado cómo la ciudadanía se muestra más tolerante con los adolescentes y jóvenes. Las investigaciones desarrolladas primero por Moon y su equipo (2000) y posteriormente por Vogel y Vogel (2003) pusieron de manifiesto que el apoyo hacia la pena de muerte se reducía sensiblemente cuando los destinatarios eran menores. De igual modo, los trabajos de Welch (2011) y de Pickett y sus colaboradores (2014) que utilizaron indicadores múltiples para medir el sentimiento punitivo encontraron un apoyo más pronunciado hacia las medidas de tolerancia cero cuando éstas se dirigían a adultos. Ciertamente, la sociedad parece valorar el desarrollo evolutivo de los jóvenes a la hora de determinar su responsabilidad penal y su potencial para la rehabilitación. En un conocido estudio, Ghetti y Redlich (2001) documentaban cómo la atribución de culpabilidad aumentaba con la edad de los delincuentes, de modo que la población asignaba una mayor responsabilidad a los adolescentes más mayores. También se han hallado diferencias relacionadas con la percepción de riesgo. Así, Salerno y su equipo (2010) mostraban cómo los agresores sexuales juveniles eran considerados menos peligrosos que sus homólogos adultos. Además, la evidencia ha revelado que los ciudadanos confían más en la eficacia de los programas de tratamiento cuando sus receptores son menores (Sundt, Cullen, Applegate y Turner, 1998). De hecho, el apoyo concedido a la rehabilitación no se ha derivado solo de preguntas abstractas, sino que ha encontrado reflejo en la disposición a costear tales medidas. En el experimento desarrollado inicialmente por Nagin et al. (2006) y replicado posteriormente por Piquero y Steinberg (2010) se observó que incluso en el supuesto de delincuentes juveniles graves, los participantes estaban dispuestos a dedicar más recursos económicos a la aplicación de programas de tratamiento (\$98.10 y \$98,49 respectivamente) que a la prolongación de las condenas privativas de libertad (\$80,97 y $\$ 84,52$ en uno y otro caso).

Aunque este trabajo ha limitado su objeto de estudio a las actitudes punitivas, sus resultados son coherentes con la literatura previa. Las diferencias entre sub-escalas revelan que las demandas de severidad no son incondicionales y que la edad de los infractores es uno de los criterios que determinan las preferencias del público. Este hallazgo no está exento de implicaciones en lo que respecta a la evolución de la justicia 
juvenil. Durante las últimas décadas, tanto en España como en otros países occidentales, se han emprendido distintas reformas que han endurecido la respuesta penal proporcionada a los menores (Applegate y Davis, 2006; Fernández y Rechea, 2006; Scott y Steinberg, 2003). A pesar de haber tenido un marcado carácter simbólico y haber sido justificadas por las exigencias de una sociedad crecientemente punitiva, este y otros trabajos (Haynes, Cares y Ruback, 2014; McGarrell y Sandys, 1996; Picket y Chiricos, 2012) sugieren que las demandas de severidad de los ciudadanos pueden haberse sobrevalorado. Al igual que la ciudadanía tiende a subestimar la dureza del sistema (Hough, Bradford, Jackson y Roberts, 2013; Hough y Roberts, 2004), el legislador puede haber magnificado sus reclamos punitivos, al menos cuando se trata de delitos cuya naturaleza no es violenta. En tales casos las penas escogidas por los participantes son similares a las que aplican los jueces, e incluso más benevolentes que ellas (Gelb, 2008; Tufts y Roberts, 2002). Sin embargo, cuando los hechos revisten especial gravedad la población se decanta por condenas más duras que las previstas legalmente (Miller y Applegate, 2015) y el apoyo otorgado a la rehabilitación se reduce notablemente (Mears et al., 2014; Sundt et al., 1998).

El segundo dato que reafirma el carácter flexible y matizado de las actitudes se desprende del análisis de conglomerados. El mismo permitió agrupar a los sujetos en tres grupos diferentes según su comportamiento en la escala de punitivismo. El primero de ellos aglutinó a las personas menos punitivas, el segundo a las moderadas y el tercero reunió al sector más punitivo. La importancia de estos resultados reside en su potencial para cuestionar que las actitudes punitivas puedan evaluarse en términos de presencia o ausencia. La obtención de distintos grupos sugiere que las demandas de severidad no son absolutas sino que obedecen a una cuestión de grado. De este modo, hay ciudadanos que apoyan o rechazan la totalidad de medidas, pero la mayoría de ellos adopta una posición graduada entre ambos extremos. El conocimiento acumulado en materia de opinión hacia la pena de muerte ha ofrecido evidencias semejantes, demostrando los problemas que entraña clasificar a los sujetos como punitivos a través de preguntas dicotómicas (si-no, favorable-desfavorable...). En el estudio desarrollado por Unnever y Cullen (2005) el 75\% de los participantes apoyó la pena de muerte cuando las alternativas de respuesta fueron si o no. Sin embargo, cuando estos mismos participantes pudieron escoger entre la pena de muerte y la cadena perpetua, el apoyo 
concedido a la primera se redujo un 22\%. En esta misma línea, los trabajos de Worthen et al. (2014) y Lambert et al. (2004) que utilizaron escalas Likert de 4 y 7 puntos mostraron que sólo una minoría de los encuestados fue totalmente favorable o desfavorable a la aplicación de la pena capital (30,87\% y 24\%, respectivamente) mientras que la generalidad de ellos se situó en algún punto intermedio entre ambos polos.

Todo ello pone de relieve la plasticidad de las actitudes hacia el delito y su control. Sin negar la existencia de posicionamientos punitivos, los datos muestran su variabilidad en función de las medidas estudiadas y de los destinatarios de dichas medidas.

\subsection{Conclusiones}

Esta investigación ha permitido validar, en el contexto español, una escala para medir las actitudes punitivas. Disponer de un instrumento válido y fiable resulta especialmente útil en un momento en el que la opinión pública ha pasado a ocupar un lugar destacado en la elaboración de las políticas públicas (Wood, 2009).

Los resultados obtenidos muestran como las iniciativas punitivas tienen una acogida desigual en la sociedad, variando notablemente de unas propuestas a otras. En general, los españoles no apoyan o rechazan su aplicación, sino que adoptan posiciones intermedias. Esta es una de las contribuciones más significativas del trabajo, pues indica que la distinción entre sujetos punitivos y no punitivos resulta insuficiente, al excluir a un sector importante de la población cuyas opiniones son moderadas.

Asimismo, los datos evidencian que las demandas de severidad no son incondicionales, reduciéndose notablemente cuando sus destinatarios son jóvenes. Este hallazgo reafirma la complejidad de las actitudes, indicando que la población no reclama medidas ejemplares de forma generalizada, reservando estas sanciones para determinados casos. 


\subsection{Limitaciones}

A pesar de sus aportaciones, la escala presenta dos limitaciones que deben ser explicadas.

La primera de ellas tiene que ver con su campo de contenido. Aun incluyendo un conjunto de indicadores que reflejan distintas vertientes del punitivismo, los mismos no agotan este constructo, cuyas manifestaciones son diversas. Futuros trabajos podrán avanzar en este sentido, incorporando indicadores adicionales que permitan conocer el posicionamiento hacia un elenco más amplio de medidas. Este estudio, como la mayoría en su ámbito, ha adoptado un enfoque de medición reflexivo (outward), entendiendo que los indicadores son el reflejo de la actitud punitiva, pero no su causa. Esta decisión ha obedecido a razones teóricas y también empíricas. No obstante será conveniente confirmar esta premisa, poniendo a prueba modelos que utilicen tanto indicadores formativos como reflexivos.

La segunda limitación se refiere al efecto del método y, más concretamente, al sesgo de aquiescencia que lleva a los encuestados a responder de forma positiva a las preguntas, con independencia de su contenido. Aunque la batería inicial incluía ítems positivos y negativos, las preguntas que finalmente conformaron la escala estaban formuladas en una misma dirección, lo cual puede haber aumentado la tendencia a responder de forma aquiescente. En un reciente experimento Pickett y Baker (2014) advertían de este fenómeno, que resultaba en un aumento artificial de la consistencia interna de las escalas. Futuras aproximaciones podrán reducir este efecto equilibrando el número de indicadores formulados en una y otra dirección.

\subsection{Implicaciones}

A pesar de las limitaciones señaladas, los resultados obtenidos han aportado evidencias de la complejidad de la opinión pública, poniendo de manifiesto que el apoyo hacia las políticas punitivas no es absoluto, sino que varía en función de las propuestas concretas y de la edad de sus destinatarios. Estos hallazgos podrán ser ampliados combinando la utilización de escalas con otras técnicas de recogida de datos, como son los casosescenario y las encuestas de valoración contingente. Este enfoque permitirá obtener una 
imagen más precisa de las preferencias del público, tanto a nivel general como en situaciones particulares.

Finalmente, debe señalarse la importancia de desarrollar investigaciones mixtas que combinen las metodologías cuantitativa y cualitativa y permitan satisfacer las demandas de conocimiento que aún hoy plantea este ámbito de estudio.

Agradecimientos: Quiero agradecer a mi directora de tesis Esther Fernández Molina su asesoramiento y orientación. Asimismo agradezco a las profesoras Cristina Rechea y Pilar Tarancón el tiempo invertido en largas y provechosas discusiones, y al investigador Casimiro Barbado su colaboración en la revisión del manuscrito. Finalmente, quiero agradecer al editor de la REIC sus valiosas observaciones que indudablemente han contribuido a mejorar la calidad científica del trabajo.

Financiación: Esta investigación se ha realizado en el marco del proyecto "Índices de Criminalidad en Castilla-La Mancha”, financiado por la Consejería de Educación y Ciencia de la Junta de Comunidades de Castilla-La Mancha (POII-2014-009-P). También ha recibido el apoyo del Programa de Formación del Profesorado Universitario (FPU) a cargo del Ministerio de Educación (AP-2012-4260).

\section{Referencias}

Aarten, P.G.M., Van Gelder, J.L., Lamet, W., Borgers, M.J. y Van der Laan, P.H. (2015). Exploring public support for suspended sentences in the Netherlands. European Journal of Criminology, 12(2), 188-207.

Adriaenssen, A. y Aertsen, I. (2015). Punitive attitudes: Towards an operationalization to measure individual punitivity in a multidimensional way. European Journal of Criminology, 12(1), 92-112.

Aizpurúa, E. (2014). Presente y futuro de la opinión pública hacia el castigo de los menores infractores. Evidencias, carencias y posibilidades. Revista Española de Investigación Criminológica, 12, art. 3, 1-29. Disponible en http://www.criminologia.net/pdf/reic/ano12-2014/a122014art3.pdf

Aizpurúa, E. y Fernández, E. (2014). ¿Procedimientos de adultos para delitos mayores? Una aproximación a la opinión pública hacia la transferencia de los menores infractores a tribunales ordinarios. Revista Electrónica de Ciencia Penal y Criminología, 16, art. 16, 1-18. Disponible en http://criminet.ugr.es/recpc/16/recpc16-16.pdf 
Allen, T.T., Trzcinscki, E. y Kubiak, S.P. (2012). Public attitudes toward juveniles who commit crimes: The relationship between assessments of adolescent development and attitudes toward severity of punishment. Crime \& Delinquency, 58(1), 78-102.

Anderson, A.L., Sample, L.L. y Cain, C.M. (2015). Residency restrictions for sex offenders: Public opinion on appropriate distances. Criminal Justice Policy Review,26(3), 262-277.

Applegate, B.K. (2001). Penal austerity: Perceived utility, desert, and public attitudes toward prison amenities. American Journal of Criminal Justice, 25(2), 253-268.

Applegate, B.K., Cullen, F.T., Turner, M.G. y Sundt, J.L. (1996). Assessing public support for three-strikes-and-you're-out laws: Global versus specific attitudes. Crime \& Delinquency, 42(4), 517-534.

Applegate, B. K. y Davis, R.K. (2006). Public views on sentencing juvenile murderers. The impact of offender, offense, and perceived maturity. Youth violence \& Juvenile Justice, 4(1), 55-74.

Baker, T., Cleary, H.M.D., Pickett, J.T. y Gertz, M.G. (2013). Crime salience and public willingness to pay for child saving and juvenile punishment. Crime \& Delinquency. Publicado online antes de impression: October 10, 2013, doi: $10.1177 / 0011128713505487$.

Baker, T., Metcalfe, C.F., Berenblum, T., Aviv, G. y Gertz, M. (2014). Examining public preferences for the allocation of resources to rehabilitative versus punitive crime policies. Criminal Justice Policy Review, 26(5), 448-462.

Baumer, E.P., Messner, S.F. y Rosenfeld, R. (2003). Explaining spatial variation in support for capital punishment: a multilevel analysis. American Journal of Sociology, 108(4), 844-875.

Bentler, P.M. (1977). Factor simplicity index and transformations. Psychometrika, 42(2), 277-295.

Butter, R., Hermanns, J. y Menger, A. (2013). Simultaneous prediction of punitive and rehabilitation-oriented attitudes towards probation: An ecological approach. Probation Journal, 60(1), 24-39.

Chen. G. y Einat, T. (2015). The relationship between criminology students and punitive attitudes. European Journal of Criminology, 12(2), 169-187.

Cochran, J.K., Boots, D.P. y Heide, K.M. (2003). Attribution styles and attitudes toward capital punishment for juveniles, the mentally incompetent, and mentally retarded. Justice Quarterly, 20(1), 65-93. 
Dawson-Edwards, C. (2008). Enfranchising convicted felons: current research on opinions toward felon voting rights. Probation and Parole: Current Issues, 46(3-4), 13-29.

Elffers, H., De Keijser, J.W., Van Koppen, P.J. y Van Haeringen, L. (2007). Newspaper juries. A field experiment concerning the effect of information on attitudes towards the criminal justice system. Journal of Experimental Criminology, 3(2), 163-182.

Evans, T.D. y Adams, M. (2003). Salvation or damnation? Religion and correctional ideology. American Journal of Criminal Justice, 28(1), 15-35.

Falco, D.L. y Turner, N.C. (2014). Examining causal attributions towards crime on support for offender rehabilitation. American Journal of Criminal Justice, 39(3), 630-641.

Fernández, E. y Rechea, C. (2006). ¿Un sistema con vocación de reforma? La Ley de Responsabilidad Penal de los Menores. Revista Española de Investigación Criminológica, 4, art. 4, 1-34. Disponible en http://www.criminologia.net/pdf/reic/ano4-2006/a42006art4.pdf

Ferrando, P.J. y Anguiano-Carrasco, C. (2010). El análisis factorial como técnica de investigación en psicología. Papeles del Psicólogo, 31(1), 18-33. Disponible en http://www.papelesdelpsicologo.es/pdf/1793.pdf

Gelb, K. (2008). More myths and misconceptions. Melbourne: Sentencing Advisory Council.

Ghetti, S. y Redlich, A. (2001). Reactions to youth crime: Perceptions of accountability and competency. Behavioral Sciences \& the Law, 19(1), 33-52.

Grasmick, H.G. y McGill, A.L. (1994). Religion, attribution style and punitiveness toward juvenile offenders. Criminology, 32(1), 23-35.

Hair, J.F., Anderson, R.E., Tatham, R.L. y Black, W.C. (2007). Análisis multivariante. $5^{\text {a }}$ Edición. Madrid: Prentice Hall.

Hamilton, C. (2014). Reconceptualizing penality. Towards a multidimensional measure of punitiveness. British Journal of Criminology, 54(1), 321-343.

Harman, H.H. (1976). Modern factor analysis. Chicago: University of Chicago Press.

Hartnagel, T.F. y Templeton, L.J. (2012). Emotions about crime and attitudes to punishment. Punishment \& Society, 14(4), 452-474.

Haynes, S.H., Cares, A.C. y Ruback, R.B. (2014). Juvenile economic sanctions. An analyses of their imposition, payment, and effect on recidivism. Criminology \& Public Policy, 13(1), 31-60. 
Hensley, C., Koscheski, M. y Tewksbury, R. (2003). College students' attitudes toward inmate programs, services, and amenities. Criminal Justice Studies, 16(4), 295-304.

Hirschfield, P.J. y Piquero, A.R. (2010). Normalization and legitimation: modeling stigmatizing attitudes toward ex-offenders. Criminology, 48(1), 27-55.

Hogan, M.J., Chiricos, T. y Gertz, M. (2005). Economic insecurity, blame, and punitive attitudes. Justice Quarterly, 22(3), 392-411.

Hohl, K., Stanko, B. y Newburn, T. (2013). The effect of the 2011 London disorder on public opinion of police and attitudes towards crime, disorder and sentencing. Policing, 7(1), 12-20.

Horn, J.L. (1965). A rationale and test for the number of factors in factor analysis. Psychometrika, 30(2), 179-185.

Hough, M., Bradford, B., Jackson, J. y Roberts, J.V. (2013). Attitudes to sentencing and trust in Justice. Exploring trends from the Crime Survey for England and Wales. London: Ministry of Justice Analytical Series.

Hough, M. y Roberts, J.V. (2004). Youth crime and youth justice. Public opinion in England and Wales. Bristol: The Policy Press.

Hu, L. y Bentler, P.M. (1999). Cutoff criteria for fit indexes in covariance structure analysis: Conventional criteria versus new alternatives. Structural Equation Modeling, 6(1), 1-55.

Hutton, N. (2005). Beyond populist punitiveness? Punishment \& Society, 7(3), 243-258.

Indermaur, D. (2009). What can we do to engender a more rational and less punitive crime policy? European Journal on Criminal Policy and Research, 15(1), 181-199.

Jerre, K. (2014). More sanctions-less prison? A research note on the severity of sanctions proposed by survey participants and how it is affected by the option to combine a prison term with other sanctions. European Journal on Criminal Policy \& Research, 20(1), 121-136.

Kornhauser, R. (2013). Reconsidering predictors of punitiveness in Australia: A test of four theories. Australian \& New Zealand Journal of Criminology, 46(2), 221-240.

Kubiak, S.P. y Allen, T. (2011). Public opinion regarding juvenile life without parole in consecutive statewide surveys. Crime \& Delinquency, 57(4), 495-515.

Lambert, E.G., Clarke, A. y Lambert, J. (2004). Reasons for supporting and opposing capital punishment in the USA: A preliminary study. Internet Journal of Criminology, 1, 1-34. Disponible en: http://www.internetjournalofcriminology.com/Clarke\%20Lambert\%20\%20Reasons\%20for\%20Supporting\%20and\%200pposing\%20Capital.pdf 
Lenz, N. (2002). “Luxuries” in prison: the relationship between amenity funding and public support. Crime \& Delinquency, 48(4), 499-525.

Lorenzo-Seva, U. (1999). Promin: A method for oblique factor rotation. Multivariate Behavioral Research, 34(3), 347-365.

Lorenzo-Seva, U. (2003). A factor simplicity index. Psychometrika, 68(1), 49-60.

Maguire, E. y Johnson, D. (2015). The structure of public opinion on crime policy: Evidence from seven Caribbean nations. Punishment \& Society, 17(4), 502-530.

Matthews, R. (2005). The myth of punitiveness. Theoretical Criminology, 9(2), 175201.

Manza, J., Brooks, C. y Uggen, C. (2004). Public Attitudes Toward Felon Disenfranchisement in the United States. Public Opinion Quarterly, 68(2), 275286.

McGarrel, E.F. y Sandys, M. (1996). The misperception of public opinion toward capital punishment: Examining the spuriousness explanation of death penalty support. The American Behavioral Scientist, 39(4), 500-513.

Mears, D.P. (2001). Getting tough with juvenile offenders. Explaining support for sanctioning youths as adults. Criminal Justice \& Behavior, 28(2), 206-226.

Mears, D.P., Mancini, C., Beaver, K.M. y Gertz, M. (2013). Housing for the "worst of the worst" inmates: Public support for supermax prisons. Crime \& Delinquency, 59(4), 587-615.

Mears, D.P., Pickett, J.T. y Mancini, C. (2014). Support for balanced juvenile justice: Assessing views about youth, rehabilitation, and punishment. Journal of Quantitative Criminology, 31(3). 459-479.

Miller, R.N. y Applegate, B.K. (2015). Adult crime, adult time? Benchmarking public views on punishing serious juveniles felons. Criminal Justice Review, 40(2), 151168.

Moon, M.M., Wright, J.P., Cullen, F.T. y Pealer, J.A. (2000). Putting kids to death: Specifying public support for juvenile capital punishment. Justice Quarterly, 17(4), 663-684.

Nagin, D.S., Piquero, A.R., Scott, E.S. y Steinberg, L. (2006). Public preferences for rehabilitation versus incarceration of juvenile offenders: Evidence from a contingent valuation survey. Crime \& Public Policy, 5(4), 301-326. 
O’Neil, K.M., Patry, M.W. y Penrod, S.D. (2004). Exploring the effects of attitudes towards the death penalty on capital sentencing veredicts. Psychology, Public Policy and Law, 10(4), 443-470.

Payne, B.K., Gainey, R.R., Triplett, R.A. y Danner, M.J.E. (2004). What drives punitive beliefs?: Demographic characteristics and justifications for sentencing. Journal of Criminal Justice, 32(3), 195-206.

Pickett, J.T. y Baker, T. (2014). The pragmatic 28merican: Empirical reality or methodological artifact? Criminology, 52(2), 195-222.

Pickett, J.T. y Chiricos, T. (2012). Controlling other people's children: Racialized views of delinquency and whites' punitive attitudes toward juvenile offenders. Criminology, 50(3), 673-710.

Pickett, J.T., Mancini, C. y Mears, D.P. (2013). Vulnerable victims, monstrous offenders, and unmanageable risk: Explaining public opinion on the social control of sex crime. Criminology, 51(3), 729-759.

Pickett, J.T., Welch, K., Chiricos, T. y Gertz, M. (2014). Racial crime stereotypes and offender juvenility: Comparing public views about youth-specific and nonyouthspecific sanctions. Race and Justice, 4(4), 381-405.

Piquero, A.R. y Steinberg, L. (2010). Public preferences for rehabilitation versus incarceration of juvenile offenders. Journal of Criminal Justice, 38(1), 1-6.

Pinaire, B., Heumann, M. y Bilotta, L. (2002). Barred from the vote: public attitudes toward the disenfranchisement of felons. Fordham Urban Law Journal, 30(5), 1519-1550.

Rial, A., Varela, J., Abalo, J. y Lévy, J.P. (2006). El análisis factorial confirmatorio. En J.P. Lévy y J. Varela (Eds.), Modelización con Estructuras de Covarianzas en Ciencias Sociales. Temas Esenciales, Avanzados y Aportaciones Especiales (pp. 119-154). A Coruña: Netbiblo.

Roberts, J.V., Crutcher, N. y Verbrugge, P. (2007). Public attitudes to sentencing in Canada: Exploring recent findings. Canadian Journal of Criminology and Criminal Justice, 49(1), 75-107.

Roberts. J.V. y Hastings, R. (2007). Public opinion and crime prevention: A review of international findings. En B. Welsh y D. Farrington (Eds.), The Oxford Handbook of Crime Prevention (pp. 487-507). Oxford: University Press.

Rogers, D.L. y Ferguson, C.J. (2011). Punishment and rehabilitation attitudes toward sex offenders versus nonsexual offenders. Journal of Aggression, Maltreatment \& Trauma, 20(4), 395-414. 
Sandys, M. Y McGarrell, E.F. (1995). Attitudes Toward Capital Punishment: Preference for the Penalty or Mere Acceptance? Journal of Research in Crime \& Delinquency, 32(2), 191-213.

Schwartz, I.M., Guo, S. y Kerbs, J.J. (1993). The impact of demographic variables on public opinion regarding juvenile justice: Implications for public policy. Crime \& Delinquency, 39(1), 5-28.

Scott, E.S. y Steinberg, L. (2003). Blaming youth. Texas Law Review, 81(3), 799-840.

Serrano, A. (2013). Actitudes hacia la pena de muerte en un país abolicionista. Un test de la teoría de los sentimientos de inseguridad. Revista Electrónica de Ciencia penal y Criminología, 15, art. 14, 1-36. Disponible en http://criminet.ugr.es/recpc/15/recpc15-14.pdf

Sharp, S.F., McGhee, M.K., Hope, T.L. y Coyne, R. (2007). Predictors of support of legislation banning juvenile executions in Oklahoma: An examination by race and sex. Justice Quarterly, 24(1), 133-155.

Shelley, T.O., Waid, C.A. y Dobbs, R.R. (2011). The influence of criminal justice major on punitive attitudes. Journal of Criminal Justice Education, 4(22), 526-545.

Sims, B. y Johnston, E. (2004). Examining public opinion about crime and justice: A statewide study. Criminal Justice Policy Review, 15(3), 270-293.

Sööt, M.L. (2013). Trust and punitive attitudes. Crime, Law and Social Change, 59(5), 537-554.

Spiranovic, C.A., Roberts, L.D., Indermaur, D., Warner, K., Gelb, K. y MacKenzie, G. (2012). Pubic preferences for sentencing purposes: What difference does offender age, criminal history and offence type make? Criminology \& Criminal Justice, 12(3), 289-306.

Sundt, J.L., Cullen, F.T., Applegate, B.K. y Turner, M.G. (1998). The tenacity of the rehabilitative ideal revisited. Have attitudes toward offender treatment changed? Criminal Justice \& Behavior, 2(4), 426-442.

Tanaka, J.S. (1993). Multifaceted conceptions of fit in structural equation models. En K. A. Bollen y J.S. Long (Eds.), Testing structural equation models (pp. 10-39). California: Sage.

Ten Berge, J.M.F. y Kiers, H.A.L. (1991). A numerical approach to the approximate and the exact minimum rank of a covariance matrix. Psychometrika, 56(2), 309315.

Timmerman, M.E. y Lorenzo-Seva, U. (2011). Dimensional assessment of ordered polytomous items with parallel analysis. Psychological Methods, 16(2), 209-220. 
Tufts, J. y Roberts, J.V. (2002). Sentencing juvenile offenders: Comparing public preferences and judicial practice. Criminal Justice Policy Review, 13(1), 46-64.

Turner, M.G., Cullen, F.T., Sundt, J.L. y Applegate, B.K. (1997). Public tolerance for community-based sanctions. The Prison Journal, 77(1), 6-26.

Tyler, T.R. y Boeckmann, R.J. (1997). Three strikes and youre out, but why? The psychology of public support for punishing rule breakers. Law \& Society Review, 31(2), 237-266.

Unnever, J.D. y Cullen, F.T. (2005). Executing the innocent and support for capital punishment: Implications for public policy. Criminology \& Public Policy, 4(1), 338.

Varona, D. (2013). Percepción y elección del castigo en España: Resultados a partir de la Encuesta Social Europea (5 ${ }^{\mathrm{a}}$ Ed.). Cuadernos de Política Criminal, 111, 145193.

Velicer, W.F. (1976). Determining the number of components from the matrix of partial correlations. Psychometrika, 41(3), 321-327.

Vogel, B.L. y Vogel, R.E. (2003). The age of death: Appraising public opinion of juvenile capital punishment. Journal of Criminal Justice, 31, 169-183.

Warner, K. y Davis, J. (2011). Using jurors to explore public attitudes to sentencing. British Journal of Criminology, 52(1), 93-112.

Welch, K. (2011). Parental status and punitiveness: Moderating effects of gender and concern about crime. Crime \& Delinquency, 57(6), 878-906.

Wood, J. (2009). Why public opinion of the criminal justice system is important. En J. Wood y T. Gannon (Eds.), Public opinion and criminal justice (pp. 33-48). Cullompton: Willan Publishing.

Worthen, M.G.F., Rodgers, F.R. y Sharp, S.F. (2014). Expanding the spectrum of attitudes toward the death penalty. Criminal Justice Review, 39(2), 160-181.

Eva Aizpurúa es becaria FPU en el Centro de Investigación de Criminología de la UCLM. Sus principales líneas de investigación comprenden la justicia juvenil y las actitudes hacia la delincuencia y su control. 